IdeAs

Idées d'Amériques

$5 \mid 2015$

Crise ou transformations du monde du travail dans les Amériques

\title{
La littérature brésilienne se dessine
}

\section{Caio César Christiano}

\section{(2) OpenEdition \\ Journals}

Édition électronique

URL : https://journals.openedition.org/ideas/975

DOI : 10.4000/ideas.975

ISSN : 1950-5701

Éditeur

Institut des Amériques

Référence électronique

Caio César Christiano, «La littérature brésilienne se dessine », IdeAs [En ligne], 5 | 2015, mis en ligne le 11 juin 2015, consulté le 19 octobre 2022. URL : http://journals.openedition.org/ideas/975 ; DOI :

https://doi.org/10.4000/ideas.975

Ce document a été généré automatiquement le 19 octobre 2022

\section{(c) (i) $\Theta$}

Creative Commons - Attribution - Pas d'Utilisation Commerciale - Pas de Modification 4.0 International - CC BY-NC-ND 4.0

https://creativecommons.org/licenses/by-nc-nd/4.0/ 


\title{
La littérature brésilienne se dessine
}

\author{
Caio César Christiano
}

1 Pour se faire remarquer, la BD a souvent besoin de briser des barrières. Il a fallu un prix Pulitzer, habituellement destinée à la "vraie littérature " pour que la saga des rats et souris Maus d'Art Spiegelman (Spiegelman A., 2012) devienne un livre digne de ce nom. Et pour se faire accepter, la BD va jusqu'à utiliser le subterfuge de l'euphémisme : il est préférable que l'adulte cultivé lise un «roman graphique » et qu'il apprécie «l'art séquentiel » plutôt qu'il aime la BD.

Dans cette optique d'un effacement de frontières entre arts apparentés - littérature et BD - qui convoite une plus grande visibilité de ce dernier, moins prestigieux, la stratégie des organisateurs du dernier Salon du livre de Paris constitue une totale réussite. Le Brésil figurait en invité d'honneur et l'infiltration d'une poignée de bédéistes parmi le très sélect groupe d'une cinquantaine d'hommes et de femmes de lettres brésiliens, dont quelques académiciens, a permis au public français et européen en général de découvrir qu'en plus de la capoeira, la samba et le carnaval, le géant sudaméricain pratique aussi la bande dessinée.

3 Comme un peu partout ailleurs, la BD brésilienne est loin d'être l'expression artistique la plus étudiée dans l'académie, mais elle subit encore une difficulté supplémentaire : en portugais brésilien le terme le plus populaire pour la BD est quadrinhos (le diminutif du mot « cadre » pour faire référence aux cases constituant normalement une histoire), ce qui nous permet de poser la question quasi philosophique : une discipline qui s'écrit au diminutif, peut-elle prétendre à une place à côté des arts majeurs?

4 Le Brésil est pourtant bien parti. Angelo Agostini, un émigré italien dessinant dans la presse brésilienne du XIX siècle figure bien parmi les pionniers de la BD mondiale (Moliterni C., Gaumier P., 1994) et c'est bien la ville de São Paulo qui accueille, en 1951, la première exposition Internationale de bandes dessinées qu'on connaisse (Moya A., 2001).

5 Un seul homme au Brésil a réussi à bâtir son empire sur l'encre de chine : Maurício de Sousa. Dans la pure tradition antropophage, chère à la culture brésilienne, il a croisé l'univers de Little Lulu de l'Américaine Marge ${ }^{1}$ avec le style du japonais Ozamu Tezuka. Sousa a ainsi créé un univers de personnages qui est immédiatement reconnaissable 
par tout brésilien de moins de 60 ans. À l'image d'un Walt Disney tropical, ses créations sont omniprésentes dans le pays et Mônica, la petite fille la plus forte du monde, est utilisée pour vendre toute sorte des produits, du coulis de tomate au dentifrice, de la pomme au shampooing. Les studios Maurício de Sousa ont pratiquement le monopole de l'univers de la BD pour enfants au Brésil, loin devant Disney, et ils ont récemment commencé à miser sur le marché pour adolescents et jeunes adultes (Beyruth D., 2013).

Mais revenons au salon du livre. Comme ils ne trouvaient pas de place dans les kiosques, dominés par Maurício de Sousa et les publications étrangères, plusieurs bédéistes brésiliens se tournent vers la bande dessinée d'auteur. La reprise de croissance des années Lula a provoqué un grand essor dans le marché éditorial et les BD brésiliennes ont commencé à trouver une place chez les libraires, dans les centres commerciaux, de plus en plus nombreux dans un pays qui s'urbanise à une vitesse impressionnante. Les éditions sont souvent luxueuses et limitées et ne sont donc accessibles qu'à une classe moyenne adulte qui a grandi avec la BD des kiosques. Le rôle des éditeurs suffisamment courageux pour publier des artistes brésiliens n'est pas à négliger et c'est dans cette position que Sandro Lobo, présent au Salon du Livre en tant que scénariste de l'album Copacabana (Lobo S., Bernardi O., 2014), a joué un rôle d'extrême importance. Son unique album à ce jour, Copacabana, montre une ville de Rio peuplée de voyous, prostituées et travestis, où le décor est davantage celui de la misère que de l'exubérance carnavalesque. Néanmoins, subsiste l'humanité de ces personnages dans un mélange de Taxi Driver de Scorsese et de la Cité de Dieu de Meirelles. L'ambiance et les traits épais du dessinateur Odyr Bernardi renvoient à la tradition de la historieta, la BD argentine, un peu plus familière au public français.

7 C'est aussi grâce à des Argentins que Marcello Quintanilha, deuxième des invités du Salon du Livre, a publié ses premiers albums en France en tant que dessinateur pour la série polar Sept balles pour Oxford (Gaú M., Zentner J., Montecarlo, 2003), scénarisé par d'autres Argentins, Zentner et Montecarlo. Son style réaliste est l'une des clés du succès de cette série de sept albums qui met en scène un détective privé qui refuse de partir à la retraite. Mais c'est dans les œuvres qu'il signe seul que se révèlent le génie et la marque de bédéiste incontournable de sa génération. Mes chers samedis (Quintanilha M., 2015) correspond peut-être à la définition de la BD brésilienne par excellence car inclassable au sein de n'importe quelle autre école (franco-belge, américaine, italienne, japonaise etc.). 


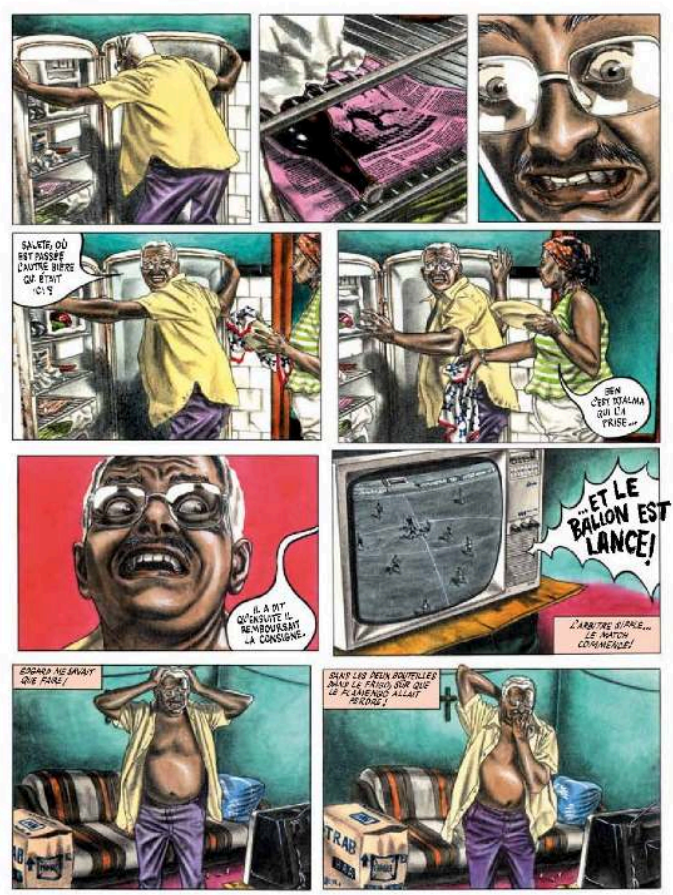

Source : Quintanilha, 2015, p. 15.

8 Ce qu'a réussi Quintanilha est formidable. On en sait plus sur le Brésil dans les cinquante pages de l'album que dans certains traités de sociologie. En choisissant de raconter plusieurs histoires qui se déroulent dans des époques différentes et dans des régions diverses du pays, Quintanilha démontre que le pays est aussi pluriel que luimême. A l'aise à la fois dans le portrait des gens des favelas tout comme dans celui des pêcheurs du Nord-Est, capable de dépeindre le football et la plage sans tomber dans les lieux-communs et la caricature, Quintanilha est en train de produire une œuvre hors pair qui le placera parmi les plus grands de la BD contemporaine, et il pourrait devenir pour la BD brésilienne ce que Sebastião Salgado est pour la photographie.

9 Les jumeaux Gabriel Bá et Fábio Moon travaillent souvent ensemble. C'est en publiant sur le marché indépendant étasunien qu'ils ont réussi à prouver que les Brésiliens étaient capables d'écrire de bons scénarios en plus de produire de beaux dessins. Le prix Eisner pour leur album Daytripper au jour le jour (Bá G., Moon F., 2012) a propulsé leur carrière internationale, et a mené à une publication en France. 


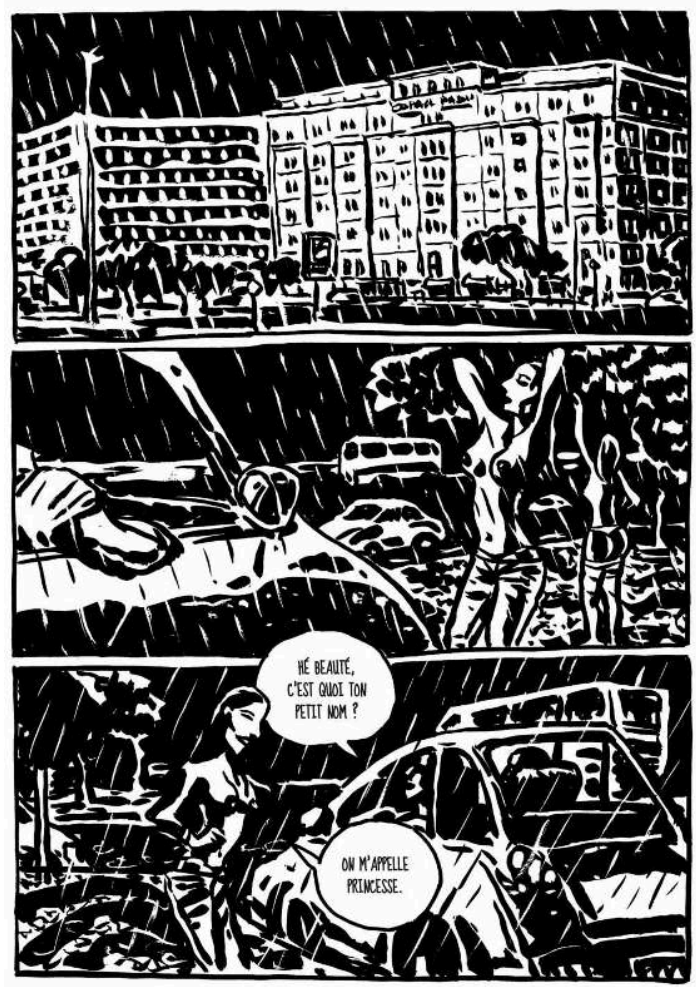

Source : Lobo S. et \& Odyr B., 2014, p. 84

10 C'est peut-être dans les récits graphiques des jumeaux que le terme hipster trouve sa meilleure définition, le côté cool à la puissance n. On y voit une São Paulo qui rêve d'être la Paris des Amériques avec ses habitants, les Paulistas, qui se prennent pour des New-yorkais. Ils sont un peu l'équivalent du réalisateur Wes Anderson de la BD. Certes, des références à des figures brésiliennes sont souvent cachées, un nom d'écrivain parci, une référence à un poète par-là, mais c'est le quotidien de l'univers personnel des jumeaux que l'on découvre plutôt que celui d'une région brésilienne spécifique. Même quand il s'agit d'adapter en BD la ville de Rio du XIX siècle, comme dans l'Aliéniste (Bá G., Moon F., 2014) d'après le conte de Machado de Assis, ou un écrivain contemporain de la région amazonienne comme dans Deux Frères (Bá G., Moon F., 2015) d'après le roman de Milton Hatoum, c'est toujours la patte branchée des frères jumeaux qu'on visualise partout. Cette idiosyncrasie de Bá et Moon ne constitue nullement un handicap, et le lecteur a toujours envie de retourner au monde hybride habité par leurs personnages.

11 De son côté, Daniel Galera, le dernier des invités du Salon que l'on peut ranger dans le club des bédéistes, a choisi de ne pas se reposer sur ses lauriers de romancier ${ }^{2}$ pour s'essayer en tant que scénariste BD. Son ouvrage Cachalot (Galera D., Coutinho R., 2012) qui compte presque 300 pages est un roman graphique à la fois émotionnel, psychologique, poétique et tragique. Six histoires qui s'entrecroisent dans un récit d'une violence inouïe. Ici, peu importe les pays par où passent les personnages, puisqu'on les voit de l'intérieur et on a souvent l'impression que même à l'intérieur, il n'y a rien. Il s'agit d'une lecture lourde comme le cétacé qui lui donne son nom et, tout comme la grande baleine, le livre est capable de plonger à des profondeurs inattendues 
où ne reste que la sensation de vide. On remarque aussi le travail du dessinateur Rafael Coutinho, essentiel pour l'unité et l'ambiance de ce roman graphique digne de ce nom.

Dans un événement comme le Salon du livre, les absents sont tout aussi remarquables que les présents. On pourrait mentionner Laerte Coutinho (le père de Rafael), figure mythique de la génération des années 1980, dont l'œuvre a connu autant d'évolutions que son propre corps. L'artiste né dans un corps masculin est depuis quelques années devenu transgenre et a opté dernièrement pour le nom de Sonia pour son activisme contre l'homophobie. Figure aussi complexe, Lourenço Mutarelli est un artiste multimédia qui pratique la $\mathrm{BD}$, le théâtre, le roman et joue même le rôle principal dans un film basé sur l'un de ses ouvrages. Ouvert à toute sorte d'espaces artistiques, son œuvre est paradoxalement marquée par une ambiance toujours claustrophobe. Leur absence est certainement expliquée par l'inexistence des traductions en langue française de leurs ouvrages. Mais il est encore temps.

Ceci ne s'applique pourtant pas à LEO. Bestseller en France, (LEO, Giraud J., 2004) avec plus d'une dizaine d'albums édités, il est certainement l'artiste BD brésilien le plus connu dans l'hexagone. Comme nul n'est prophète en son pays, LEO n'a publié qu'un seul album dans sa terre natale. S'il n'a pas été invité, ce fut certainement faute d'avoir très peu publié en langue portugaise et d'être peut-être méconnu des organisateurs. Comme l'autre bestseller brésilien, Paulo Coelho a lui aussi, finalement manqué le rendez-vous à cause d'un problème aérien. Certains ont comparé la fête de l'écriture brésilienne, sans la présence des deux Brésiliens les plus lus en France, à une visite dans un certain petit village gaulois de l'an 50 av. J.-C. lorsqu'Astérix et Obélix ne s'y trouvaient pas.

Il y bien d'autres Brésiliens qui publient des BD en France comme André Diniz, avec ses récits sociaux et ses portraits de la favela (Diniz A., 2012), Wander Antunes, raconteur habile, capable d'aller du far-West (Antunes W., Taborda W., 2005) aux navires pirates (Antunes W., Tirso, 2005) à la manière des vieux films d'aventures, et Gustavo Duarte dont l'album Monstres (Duarte G., 2012) a récemment été publié en France sans traduction (sauf le titre), car il raconte ses histoires de tarasques hideuses et de bêtes fabuleuses sans paroles. Chacun apporte une vision distincte de la complexité brésilienne qui vaut toujours le détour. Après ses récents échecs au football, terrain où il a un jour régné, il est peut-être temps pour le Brésil de penser à devenir le pays de la BD.

\section{BIBLIOGRAPHIE}

Antunes, Wander et Taborda, Walther, Big Bill est mort, Paris, Paquet, 2005.

Antunes, Wander et Cons, Tirso, L'œil du diable, Paris, Paquet, 2005

Bá, Gabriel et Fabio Moon, Daytripper au jour le jour [éd. orig. Daytripper, New York, DC Comics], Paris, Urban Comics, 2012 [2011]. 
Bá, Gabriel et Moon, Fabio, Deux frères [éd. orig. Dois irmãos, São Paulo, Companhia das Letras], Paris, Urban Comics, 2015 [2011].

Bá, Gabriel et Moon, Fabio, L'aliéniste [éd. orig. O Alienista, São Paulo, Agir], Paris, Urban Comics, 2014 [2007].

Beyruth, Danilo, Astronaute - Au cœur du Magnétar, Paris, Panini, 2013

Diniz, André, Photo de la Favela [éd. orig. Morro da favela, São Paulo, Barba Negra/Leya], Vincennes, Des ronds dans l'o Éditions, 2012 [2011].

Duarte, Gustavo, Monstres [éd. orig. Monstros, São Paulo, Companhia das Letras], Paris, Paquet, 2015 [2012].

Galera, Daniel et Coutinho, Rafael, Cachalot [éd. orig. Cachalote, São Paulo, Companhia das Letras], Paris, Cambourakis, 2012 [2010].

Galera, Daniel, La barbe ensanglantée [éd. orig. A barba ensopada de sangue, São Paulo, Companhia das Letras], Paris, Gallimard, 2015 [2012].

Galera, Daniel, Paluche [éd. orig. Mãos de cavalo, São Paulo, Companhia das Letras], Paris, Gallimard, 2010 [2006].

Gaú, Marcello, Jorge Zentner et Montecarlo, 7 balles pour Oxford, tome 1: La Promesse, Paris, Lombard, 2003.

LEO et Giraud, Jean, Aldébaran : L'intégrale, Paris, Dargaud, 2004.

Lobo, Sandro et Bernardi, Odyr, Copacabana [éd. orig. Copacabana, Rio De Janeiro, Desiderata], Paris, Warum, 2014 [2009].

Moya, Álvaro de, Anos 50: 50 anos, São Paulo, Ópera Graphica, 2001.

Moliterni, Claude et Gaumier, Patrick, Dictionnaire mondial de la Bande Dessinée, Paris, Larousse, 1994.

Quintanilha, Marcello, Mes chers samedis [éd. orig. Sábado dos meus amores, São Paulo, Conrad], Paris, Çà et Là, 2015 [2009].

Spiegelman, Art, L'intégrale, Maus [éd. orig. The Complete Maus, 25th Anniversary Edition, New York, Pantheon Books], Paris, Flammarion, 2012 [1996].

\section{NOTES}

1. Marge est le pseudonyme de l'artiste Marjorie Henderson Buell, l'une des rares femmes artistes de BD dans les années 1930. Très peu connue en France, son personnage phare a connu quelques publications sous la traduction de Petite Lulu.

2. Ses romans Paluche et La barbe ensanglantée (Galera D., 2010 ; 2015) sont parus en France chez Gallimard dans la traduction de Maryvonne Lapouge-Pettorelli. 


\section{AUTEUR}

\section{CAIO CÉSAR CHRISTIANO}

Caio César Christiano est lecteur de l'Ambassade du Brésil à l'Université Blaise-Pascal, Clermont II. Docteur en linguistique, il a enseigné à l'Université de Poitiers pendant 3 ans et à l'Institut de sciences politiques de Paris pendant 6 ans. Ses champs de recherche sont la linguistique de corpus, l'enseignement des langues étrangères, la traduction audio-visuelle et l'analyse du discours.

Parallèlement à son activité universitaire, il est traducteur et participe régulièrement à des tables-rondes et conférences sur la BD lusophone. En 2004, il a créé le « Gibiblog », le site internet pionnier dans la promotion et analyse de la BD brésilienne. caiochristiano@gmail.com 